[0212-7199 (2006) 23: 10; pp 465-474] ANALES DE MEDICINA INTERNA Copyright (C) 2006 ARAN EDICIONES, S.L.

AN. MED. INTERNA (Madrid) Vol. 23, N. ${ }^{\circ} 10$, pp. 465-474, 2006

\title{
Perfiles de asociación de factores de riesgo o enfermedad cardiovascular en población española de alto riesgo de 2.264 pacientes
}

\author{
B. EBRÍ TORNÉ, I. EBRÍ VERDE, J. SARDAÑA FERRER, A. PORTOLÉS SUSO, \\ C. SUÁREZ FERNÁNDEZ', EN REPRESENTACIÓN DEL EQUIPO DE \\ INVESTIGADORES DEL GRUPO CIFARC (GRUPO DE RIESGO VASCULAR DE \\ LA SEMI)
}

\author{
Hospital Universitario Miguel Servet. Zaragoza. ${ }^{1}$ Hospital Universitario de La Princesa. \\ Madrid
}

\begin{abstract}
ASOCIATION PATTERNS OF RISK FACTORS OR CARDIOVASCULAR DISEASE, IN A HIGH RISK SPANISH POPULATION OF 2,264 PATIENTS
\end{abstract}

\section{RESUMEN}

Introducción, fundamentos y objetivos: El objetivo de este estudio es presentar los diversos perfiles asociativos entre factores de riesgo cardiovascular y lesión de órganos diana de la HTA, así como establecer las posibles diferencias entre sexos y por edades y comparar nuestros hallazgos con los reseñados en la bibliografía.

Material y métodos: Estudio transversal de ámbito nacional con 2.264 pacientes consecutivos de las Consultas Externas de Medicina Interna, mayores de 18 años y con riesgo cardiovascular alto o muy alto, procedentes del Estudio CIFARC del Grupo de Riesgo Vascular de la SEMI. El tratamiento estadístico (Chi cuadrado, ANOVA y regresión líneal de Pearson) se ha realizado mediante el programa STATISTIX.

Resultados y discusión: Hemos obtenido correlaciones estadísticamente significativas, entre: Colesterol total elevado y colesterol LDL, colesterol HDL bajo y tabaquismo. Colesterol LDL con Antecedentes Familiares Precoces de eventos cardiovasculares (AFP) y cardiopatía isquémica. Colesterol HDL bajo con AFP. Tabaquismo con AFP. HVI con elevaciones de proteinuria y creatinina, ateromatosis, retinopatía I-II, cardiopatía isquémica y nefropatía diabética. Cardiopatía isquémica con HVI, nefropatía diabética, enfermedad vascular periférica (EVP) y edad. Proteinuria con elevaciones de creatinina, ateromatosis, retinopatía I-II, nefropatía y retinopatía grave. Retinopatía I-II con elevaciones de creatinina, ateromatosis, nefropatía, EVP, y retinopatía grave. Elevaciones de creatinina con colesterol HDL descendido, proteinuria, ateromatosis, retinopatía I-II, nefropatía y EVP. $A F P$ con la edad inversamente: Los pacientes con AFP acuden una media de tres años antes a consultas que los pacientes que no los presentan. Ateromatosis con HVI, elevaciones de proteinuria y creatinina, retinopatía I-II, enfermedad cerebrovascular, nefropatía, EVP y edad. EVP con creatinina elevada, ateromatosis, retinopatía I-II y cardiopatía isquémica. Enfermedad cerebrovascular con ateromatosis, edad y peso. Retinopatía grave con proteinuria, nefropatía y retinopatía I-II nefropatía diabética con elevaciones de proteinuria y creatinina, ateromatosis, retinopatía I-II, cardiopatía isquémica y retinopatía grave. Edad con creatinina elevada, ateromatosis, cardiopatía isquémica y enfermedad cerebrovascular. Respecto al sexo encontramos diferencias en casi todas las variables.

Conclusiones: Existen diferentes perfiles asociativos entre las variables estudiadas.

PALABRAS CLAVE: Población española de 2.264 pacientes. Estudio transversal. Perfiles de asociación de factores de riesgo o enfermedad cardiovascular.

\section{ABSTRACT}

Introduction, bases and aims: The aim of this study is to present the different association patterns between cardiovascular disease risk factors and target organs lesion due to arterial hypertension $(A H)$, and to establish the possible differences by gender and age, and compare our findings with the existing references in the medical literature.

Material and methods: Nationwide croo-sectional study with 2264 consecutive patients in the Outpatient Clinic of Internal Medicine 18 years of age or older and with high cardiovascular risk taken from the CIFARC (Integral Control of High Risk Cardiovascular Factors) study run by the SEMI (Spanish Internal Medicine Association) cardiovascular Risk Group.

Results and discussion: We have obtained statistically significant correlations between: Raised levels of total cholesterol and LDL cholesterol, low HDL cholesterol and smoking habit. LDL cholesterol with Family History of early cardiovascular events (hereinafter FH) and ischemic cardiopathy. Low HDL cholesterol with FH. Smoking habit with FH. Left ventricular hypertrophy $(\mathrm{LVH})$ with raised levels of proteinuria and creatinine, atheromatosis, retinopathy I-II, ischemic cardiopathy, and diabetic nephropathy. Ischemic cardiopathy with $\mathrm{LVH}$, diabetic nephropathy, peripheral vascular disease $(P V D)$ and age. Proteinuria with raised levels of creatinine, atheromatosis, retinopathy I-II, nephropathy and severe retinopathy. Retinopathy I-II with raised levels of creatinine, atheromatosis, nephropathy, $P V D$ and severe retinopathy. Raised levels of creatinine with reduced levels of HDL cholesterol, proteinuria, atheromatosis, retinopathy I-II, nephropathy and PVD. FH inversely with age: patients with FH consult a doctor an average of three years earlier than patients who do not experience FH. Atheromatosis with $\mathrm{LVH}$, raised levels of proteinuria and creatinine, retinopathy I-II, cerebrovascular disease, nephropathy, PVD and age. PVD with raised levels of creatinine, atheromatosis, retinopathy I-II, and ischemic cardiopathy. Cerebrovascular disease with atheromatosis, age and weight. Severe retinopathy with proteinuria, nephropathy, and retinopathy I-II. Diabetic nephropathy with raised levels of proteinuria and creatinine, atheromatosis, retinopathy I-II, ischemic cardiopathy, and severe retinopathy. Age with raised creatinine, atheromatosis, ischemic cardiopathy, and cerebrovascular disease. With respect to gender, we found differences in most of the studied variables.

Conclusions: There exist different association patterns between the studied variables.

KEY WORDS: Spanish population of 2,264 patients. Cross-sectional study. Asociation patterns of risk factors or cardiovascular disease.

Ebrí Torné B, Ebrí Verde I, Sardaña Ferrer J, Portolés Suso A, Suárez Fernández C, en representación del equipo de investigadores del Grupo CIFARC (Grupo de Riesgo Vascular de la SEMI). Perfiles de asociación de factores de riesgo o enfermedad cardiovascular en población española de alto riesgo de 2.264 pacientes. An Med Interna (Madrid) 2006; 23: 465-474. 


\section{INTRODUCCIÓN}

La cardiopatía isquémica continua siendo la primera causa individual de muerte en varones y la tercera en mujeres, siendo la causa del $10 \%$ del total de muertes producidas en 1997 , respectivamente (1-3). La incidencia de infarto de miocardio (IAM), número de casos nuevos/100.000 habitantes y año en la población española de 35 a 64 años es de las más bajas del mundo y parece estar estabilizada en los últimos quince años (4-5). La mayoría de muertes se producen después de los 64 años. La enfermedad cardiovascular es responsable de un tercio del global de muertes en el mundo (6,7). Alrededor del $3 \%$ de los pacientes con IAM tienen antecedentes de 2 o más factores de riesgo clásico como también hay un $18 \%$ que no tienen ninguno conocido (8). Dada la confluencia de factores de riesgo cardiovascular y la impotencia de actuar en su prevención, resulta interesante conocer los diversos perfiles asociativos de factores con las diversas patologías cardiovasculares, para actuar en su prevención. La enfermedad cardiovascular constituye uno de los principales problemas de salud de la población española, pudiéndose anticipar que su impacto sanitario crecerá en los próximos años como consecuencia de un envejecimiento de la población y la consolidación de la transición epidemiológica que lleva aparejada un importante predominio de las enfermedades crónicas sobre las ayudas. A partir de la información presentada en el Informe SEA de 2003, se producen en España cerca de 132.000 muertes y más de cinco millones de estancias hospitalarias por enfermedades cardiovasculares al año. Son por ello la primera causa de muerte y de hospitalización en la población española. La mitad de la población presenta valores de colesterol total superiores de más de $200 \mathrm{mg} / \mathrm{dl}$. El porcentaje de población con hipertensión arterial es del $40 \%$. Este porcentaje aumenta con la edad y alcanza el $68 \%$ en las personas de 60 y más años. Sólo el $16 \%$ de la población hipertensa española tiene controlada la presión arterial de forma óptima. La hipertensión arterial puede dar cuenta del $40 \%$ de las muertes por ictus. El $36 \%$ de los españoles fuma. Los jóvenes españoles tienen una de las prevalencias de tabaquismo más alta de Europa. El tabaco produce 52.000 muertes al año en España. En el conjunto de la población española de 2 a 24 años, el porcentaje de sobrepeso es del 21,4\% y el de obesidad es del 5,8\%. Ya en la actualidad 28.000 muertes cada año son atribuibles a la obesidad en España. El $46 \%$ de la población de 16 y más años declara no realizar actividad física alguna durante el tiempo libre. El porcentaje de población con diabetes se estima en un $6 \%$ para los grupos de edad de 30 a 65 años, y del 10\% para los 30 a 89 años. La atención a las enfermedades cardiovasculares fue responsable en 1993 del 15\% de los costes sanitarios totales.

El objetivo de este trabajo es presentar los diversos perfiles asociativos entre factores de riesgo cardiovascular y lesión de órganos diana de la HTA, que hemos encontrado en una población española de alto riesgo de 2.264 individuos, así como establecer las posibles diferencias entre sexos y por edades. Así mismo comparar nuestros hallazgos con la bibliografía.

\section{MATERIAL Y MÉTODOS}

La casuística de 2.264 pacientes procede del Estudio CYFARC (Grupo de Riesgo Vascular de la SEMI). Los pacientes mayores de 18 años proceden de toda la geografía española; y acudieron a Consultas Externas de Medicina
Interna, por la existencia de factores de riesgo o enfermedad cardiovascular. $\mathrm{Su}$ riesgo es alto o muy alto. Según la Escala de Framingham es superior al 20\% a los 10 años; 54\% son varones y el $46 \%$ mujeres. La edad media es de $66 \pm 11,5$ años. Se han estudiado y correlacionado estadísticamente las siguientes variables: sexo, IMC (Índice de Masa Corporal), peso, talla, edad, cifras de colesterol total de más de 250 $\mathrm{mg} / \mathrm{dl}$, cifras de colesterol LDL superior a $130 \mathrm{mg} / \mathrm{dl}$, cifras de colesterol HDL inferiores a $40 \mathrm{mg} / \mathrm{dl}$, HVI (hipertrofia ventricular izquierda), niveles de creatinina de 1,2 a $2 \mathrm{mg} / \mathrm{dl}$, retinopatía I-II, retinopatía grave, tabaquismo, proteinuria de más de $300 \mathrm{mg} / \mathrm{dl}$, nefropatía diabética o insuficiencia renal, ateromatosis, cardiopatía isquémica, enfermedad cerebrovascular y enfermedad vascular periférica. Para el tratamiento estadístico se ha utilizado el programa STATISTIX, realizándose $\mathrm{Chi}^{2}$, análisis de varianza (Anova) y regresión líneal de Pearson.

\section{RESULTADOS}

Exponemos en las tablas adjuntas las relaciones de cada variable con el resto, en cada sexo y de forma global. Cuando existen asociaciones entre variables, éstas son significativas estadísticamente a $\mathrm{p}<0,05$.

Cuando las variables son categóricas, y existe asociación entre ellas, se aprecia un incremento de porcentaje mayor de la variable observada respecto a lo esperado, hecho que expresa su significación estadística, y que se detalla en las tablas. Cuando las variables son categóricas y continuas se analizan las diferencias de medias y si las variables son continuas, el coeficiente de correlación.

En honor a la brevedad y por razón de espacio, como muestrario de este trabajo presentamos únicamente un total cinco tablas que expresan ya directamente, por su significación estadística, el perfil de asociación de las diferentes variables analizadas. Se exponen no obstante en este apartado, los resultados del perfil asociativo de todas las variables que componen este estudio, y que forman en definitiva los diferentes perfiles básicos de riesgo cardiovascular. Las tablas adjuntas son las siguientes:

Número I: colesterol total (más de $250 \mathrm{mg} / \mathrm{dl}$ ); número II: tabaquismo; número III: cardiopatía isquémica; número IV: ateromatosis; número $\mathrm{V}$ : edad.

\section{COLESTEROL TOTAL DE MÁS DE $250 \mathrm{mg} / \mathrm{dl}$ (Tabla I)}

- Se encuentra correlacionado en cada sexo y en el total de hombres y mujeres, con el colesterol LDL > $130 \mathrm{mg} / \mathrm{dl}$, colesterol HDL $<40 \mathrm{mg} / \mathrm{dl}$, y tabaquismo. En varones con AFP de eventos cardiovasculares, cardiopatía isquémica, peso, edad, e IMC. Con la edad es a la inversa, de tal manera que las personas de menor edad son las que tienen el colesterol más alto, es decir la población con colesterol total de más de $250 \mathrm{mg} / \mathrm{dl}$, tiene una edad de 3,5 años de media más joven que los que tienen el colesterol total de $<$ de $250 \mathrm{mg} / \mathrm{dl}$. Sin embargo su peso es mayor: $2,25 \mathrm{~kg}$, e indica una masa corporal más elevada: 0,62 de media. Correlacionado sólo en varones con retinopatía I-II, y enfermedad cerebrovascular, con ésta de forma negativa o inversa. Un $28 \%$ varones y $26 \%$ mujeres tienen cifras de colesterol total elevado. 
TABLA I

PERFILES DE ASOCIACIÓN DE LA VARIABLE COLESTEROL TOTAL

\begin{tabular}{lccc}
\hline Colesterol total $>250 \mathrm{mg} / \mathrm{dl}$ & & Varones & Mujeres \\
\hline Sexo & + & $\mathrm{ns}$ & $\mathrm{ns}$ \\
$\mathrm{LDL}>130 \mathrm{mg} / \mathrm{dl}$ & $* *$ & $* * *$ & $* * *$ \\
$\mathrm{HDL}<40 \mathrm{mg} / \mathrm{dl}$ & $* *$ & $*$ & $*$ \\
Tabaquismo & $* *$ & $* *$ & $*$ \\
$\mathrm{HVI}$ & $\mathrm{ns}$ & $\mathrm{ns}$ & $\mathrm{ns}$ \\
Proteinuria $>300 \mathrm{mg} / 24$ horas & $\mathrm{ns}$ & $\mathrm{ns}$ & $\mathrm{ns}$ \\
Creatinina 1,2-2,0 mg/dl & $\mathrm{ns}$ & $\mathrm{ns}$ & $\mathrm{ns}$ \\
Ateromatosis & $\mathrm{ns}$ & $\mathrm{ns}$ & $\mathrm{ns}$ \\
Retinopatía I-Il & $\mathrm{ns}$ & $*$ & $\mathrm{~ns}$ \\
AFP & $*$ & $*$ & $\mathrm{~ns}$ \\
Cardiopatia isquémica & $* *$ & $* *$ & $\mathrm{~ns}$ \\
Enf. cerebrovascular & + & $*$ & $\mathrm{~ns}$ \\
Nefropatia diabética & $\mathrm{ns}$ & $*$ & $\mathrm{~ns}$ \\
Enf. vascular periférica & $\mathrm{ns}$ & $\mathrm{ns}$ & $\mathrm{ns}$ \\
Retinopatía grave & $\mathrm{ns}$ & $\mathrm{ns}$ & $\mathrm{ns}$ \\
Peso & $* *$ & $* *$ & + \\
Talla & $\mathrm{ns}$ & $\mathrm{ns}$ & $\mathrm{ns}$ \\
Edad & $* * *$ & $* * *$ & $\mathrm{~ns}$ \\
IMC & $* *$ & $*$ & $\mathrm{~ns}$ \\
\hline
\end{tabular}

ns: $p>0,100 ;+: p<0,100 ;{ }^{*}: p<0,050 ;{ }^{* *}: p<0,010 ;{ }^{* * *}: p<0,001$

\section{COLESTEROL LDL DE MÁS DE $130 \mathrm{mg} / \mathrm{dl}$}

- Correlacionado en cada sexo, y en el total de la casuística, hombres y mujeres, con cifras de colesterol total elevado, colesterol HDL bajo y tabaquismo. Con la edad se encuentra correlacionado de forma inversa, es decir la población que posee cifras de LDL más altas presenta una media de edad de 3,2 años más joven en varones, y de 1,91 años en mujeres. Correlacionado en varones y serie total de hombres y mujeres, con AFP de eventos cardiovasculares, y talla: $1,12 \mathrm{~cm}$ más de media en varones, y $1,15 \mathrm{~cm}$ más en serie total conjunta, en la población que posee mayores cifras de colesterol LDL. Correlacionado en mujeres y serie global, con la cardiopatía isquémica. En varones con el peso: 1,66 kg más de media en la población que posee un colesterol LDL elevado. Un $32 \%$ de varones y un $26 \%$ de mujeres tienen cifras de colesterol LDL por encima de $130 \mathrm{mg} / \mathrm{dl}$.

\section{COLESTEROL HDL DE MENOS DE $40 \mathrm{MG} / D L$}

- Correlacionado en cada sexo y en el total, hombres y mujeres, con cifras de colesterol total elevado, tabaquismo y niveles elevados de creatinina. Así mismo con la talla con una diferencia media mayor de $3,43 \mathrm{~cm}$ en serie global, $1,96 \mathrm{~cm}$ en varones, y 2,51 en mujeres, respecto a la población que no tiene el colesterol HDL bajo.Correlacionado en varones y en la serie total con AFP. Correlacionado en mujeres, y en serie total con el peso, con unas diferencias de $2,59 \mathrm{~kg}$ más en serie total y de $3,07 \mathrm{~kg}$ en mujeres, respecto a la población que tiene el colesterol HDL más alto de $40 \mathrm{mg} / \mathrm{dl}$. En mujeres con la enfermedad cerebrovascular y además de forma inversa.Un $13 \%$ de varones y un $8 \%$ mujeres, tienen cifras de colesterol HDL por debajo de $40 \mathrm{mg} / \mathrm{dl}$.

\section{TABAQUISMO (Tabla II)}

- Correlacionado en cada sexo y en el total, hombres y mujeres, con cifras elevadas de colesterol total y de LDL, cifras bajas de HDL. Así mismo con la talla y la edad. Con esta última de forma inversa, de tal manera que la población fumadora es más joven que la no fumadora, en la serie global con 6,11 años de media menos; en hombres con 5,14 años, y en mujeres con 7,1 años. Correlacionado en varones y en la serie global con AFP de eventos cardiovasculares. También con IMC aunque de forma inversa. En la serie global con niveles incrementados de creatinina, peso, sexo y enfermedad vascular periférica. En hombres con enfermedad cerebrovascular y además de forma inversa. Un $15 \%$ de varones y un 5\% de mujeres de la casuística se declaran fumadores.

\begin{tabular}{lccc}
\multicolumn{4}{c}{ TABLA II } \\
\multicolumn{3}{c}{ PERFILES DE ASOCIACIÓN DE LA VARIABLE TABAQUISMO } \\
\hline Tabaquismo & & Varones & Mujeres \\
\hline Sexo & $* * *$ & $\mathrm{~ns}$ & $\mathrm{~ns}$ \\
Colesterol total $>250 \mathrm{mg} / \mathrm{dl}$ & $* *$ & $* *$ & $*$ \\
$\mathrm{LDL}>130 \mathrm{mg} / \mathrm{dl}$ & $* * *$ & $* *$ & $* *$ \\
$\mathrm{HDL}<40 \mathrm{mg} / \mathrm{dl}$ & $* * *$ & $* * *$ & $* *$ \\
$\mathrm{HVI}$ & $\mathrm{ns}$ & $\mathrm{ns}$ & $\mathrm{ns}$ \\
Proteinuria $>300 \mathrm{mg} / 24$ horas & + & $\mathrm{ns}$ & $\mathrm{ns}$ \\
Creatinina $1,2-2,0 \mathrm{mg} / \mathrm{dl}$ & $* *$ & + & $\mathrm{ns}$ \\
Ateromatosis & $\mathrm{ns}$ & $\mathrm{ns}$ & $\mathrm{ns}$ \\
Retinopatía I-II & $\mathrm{ns}$ & $\mathrm{ns}$ & $\mathrm{ns}$ \\
AFP & $* *$ & $* * *$ & $\mathrm{~ns}$ \\
Cardiopatia isquémica & $\mathrm{ns}$ & $\mathrm{ns}$ & $\mathrm{ns}$ \\
Enf. cerebrovascular & $\mathrm{ns}$ & $* *$ & $\mathrm{~ns}$ \\
Nefropatía diabética & $\mathrm{ns}$ & $\mathrm{ns}$ & $\mathrm{ns}$ \\
Enf. vascular periférica & $* *$ & $\mathrm{~ns}$ & $\mathrm{~ns}$ \\
Retinopatía grave & + & + & $\mathrm{ns}$ \\
Peso & $* *$ & $\mathrm{~ns}$ & $\mathrm{~ns}$ \\
Talla & $* * *$ & $* * *$ & $* * *$ \\
Edad & $* * *$ & $* * *$ & $* * *$ \\
IMC & $* * *$ & $*$ & $\mathrm{~ns}$ \\
\hline
\end{tabular}

ns: $p>0,100 ;+: p<0,100 ;{ }^{*}: p<0,050 ;{ }^{* *}: p<0,010 ;{ }^{* *}: p<0,001$.

\section{HIPERTROFIA VENTRICULAR IZQUIERDA (HVI)}

- Correlacionada en cada sexo y en el total, hombres y mujeres, con cifras elevadas de proteinuria en 24 horas, niveles elevados de creatinina, ateromatosis, retinopatía I-II, cardiopatía isquémica y nefropatía diabética.En varones y serie global con AFP de eventos cardiovasculares, enfermedad vascular periférica y retinopatía grave.En serie total con IMC. Existen diferencias entre sexos de edad, de tal manera que las pacientes con HVI tienen una diferencia de 2,38 años más de media que los varones con HVI. Un $21 \%$ de varones y un $13 \%$ de mujeres presentan HVI. 


\section{CARDIOPATÍA ISQUÉMICA (Tabla III)}

- Correlacionada en serie global y en cada sexo con HVI, nefropatía diabética, enfermedad vascular periférica y edad. En varones y en serie global con cifras elevadas de colesterol total, niveles elevados de creatinina, y ateromatosis. En serie global con retinopatía grave. Correlacionada sólo en varones con talla y de forma inversa. En mujeres con peso e IMC. En serie global y mujeres con niveles elevados de colesterol LDL. Un $22 \%$ de varones y un $15 \%$ de mujeres presentan cardiopatía isquémica.

\section{TABLA III}

PERFILES DE ASOCIACIÓN DE LA VARIABLE CARDIOPATÍA ISQUÉMICA

\begin{tabular}{lccc}
\hline Cardiopatía isquémica & & Varones & Mujeres \\
\hline Sexo & $* *$ & $\mathrm{~ns}$ & $\mathrm{~ns}$ \\
Colesterol total $>250 \mathrm{mg} / \mathrm{dl}$ & $* *$ & $* *$ & $\mathrm{~ns}$ \\
$\mathrm{LDL}>130 \mathrm{mg} / \mathrm{dl}$ & $* *$ & + & $* *$ \\
$\mathrm{HDL}<40 \mathrm{mg} / \mathrm{dl}$ & $\mathrm{ns}$ & $\mathrm{ns}$ & $\mathrm{ns}$ \\
Tabaquismo & $\mathrm{ns}$ & $\mathrm{ns}$ & $\mathrm{ns}$ \\
$\mathrm{HVI}$ & $* * *$ & $* * *$ & $* * *$ \\
Proteinuria $>300 \mathrm{mg} / 24$ horas & + & $\mathrm{ns}$ & $\mathrm{ns}$ \\
Creatinina 1,2-2,0 mg/dl & $* *$ & $*$ & $\mathrm{~ns}$ \\
Ateromatosis & $* *$ & $* *$ & $\mathrm{~ns}$ \\
Retinopatía I-Il & $\mathrm{ns}$ & $\mathrm{ns}$ & $\mathrm{ns}$ \\
AFP & $\mathrm{ns}$ & $\mathrm{ns}$ & + \\
Enf. cerebrovascular & $\mathrm{ns}$ & $\mathrm{ns}$ & $\mathrm{ns}$ \\
Nefropatía diabética & $* * *$ & $* * *$ & $* * *$ \\
Enf. vascular periférica & $* * *$ & $* * *$ & $*$ \\
Retinopatía grave & $*$ & + & $\mathrm{ns}$ \\
Peso & $\mathrm{ns}$ & + & $*$ \\
Talla & $\mathrm{ns}$ & $*$ & $\mathrm{~ns}$ \\
Edad & $* * *$ & $* * *$ & $* * *$ \\
IMC & $\mathrm{ns}$ & $\mathrm{ns}$ & $*$ \\
\hline
\end{tabular}

ns: $p>0,100 ;+: p<0,100 ;{ }^{*}: p<0,050 ;{ }^{* *}: p<0,010 ; *^{* *}: p<0,001$.

\section{PROTEINURIA DE MÁS DE 300 mg/24 HORAS}

- Correlacionada en la serie global como en cada sexo por separado con niveles elevados de creatinina, ateromatosis, retinopatía I-II, nefropatía diabética, retinopatía grave.En varones y en serie global con enfermedad vascular periférica. En la serie global con talla. Correlacionada sólo en varones con AFP de eventos cardiovasculares. Sólo en mujeres con cifras de colesterol HDL bajo.Un 10\% de varones y un 5\% de mujeres presentan proteinuria.

\section{RETINOPATÍA I-II}

- Correlacionada en la serie global como en cada sexo por separado con HVI, niveles elevados de proteinuria y creatinina, ateromatosis, nefropatía diabética, enfermedad vascular periférica y retinopatía grave. En varones y en serie global con AFP y enfermedad cerebrovascular, y de forma inversa.
Correlacionada sólo en varones con cifras altas de colesterol total e IMC. Un 10\% de varones y un 9\% de mujeres presentan retinopatía I-II.

\section{NIVELES DE CREATININA ELEVADOS: 1,2-2 mg/dl}

- Correlacionados en serie global y en cada sexo con cifras bajas de colesterol HDL, HVI; proteinuria , ateromatosis, retinopatía I-II, nefropatía diabética,, enfermedad vascular periférica y edad. En varones y en serie global con AFP, cardiopatía isquémica, retinopatía grave e IMC, con este último parámetro de forma inversa. En la serie global con la talla. En varones con tabaquismo, y peso, con este último parámetro de forma inversa. Un $10 \%$ de varones y un $8 \%$ de mujeres presentan niveles de creatinina elevados.

\section{AFP DE EVENTOS CARDIOVASCULARES}

- Correlacionados en la serie global y en cada sexo con la edad, y de forma inversa. Es decir los pacientes que tienen AFP de eventos cardiovasculares acuden una media de tres años antes a consulta médica que los pacientes que no presentan dichos antecedentes. Correlacionados en varones y en serie global con cifras elevadas de colesterol total y LDL, con cifras bajas de HDL, tabaquismo, HVI, niveles elevados de creatinina, retinopatía I-II y talla. En varones con retinopatía grave y proteinuria. En serie global y en mujeres con enfermedad vascular periférica. Un 6,4\% de varones y un 5,3\% de mujeres presentan AFP de eventos cardiovasculares.

\section{ATEROMATOSIS (Tabla IV)}

- Correlacionada en la serie global yen cada sexo por separado con HVI, niveles elevados de proteinuria y creatinina, reti-

\section{TABLA IV}

PERFILES DE ASOCIACIÓN DE LA VARIABLE ATEROMATOSIS

\begin{tabular}{lccc}
\hline Ateromatosis & & Varones & Mujeres \\
\hline Sexo & $*$ & $\mathrm{~ns}$ & $\mathrm{~ns}$ \\
Colesterol total $>250 \mathrm{mg} / \mathrm{dl}$ & $\mathrm{ns}$ & $\mathrm{ns}$ & $\mathrm{ns}$ \\
$\mathrm{LDL}>130 \mathrm{mg} / \mathrm{dl}$ & $\mathrm{ns}$ & $\mathrm{ns}$ & $\mathrm{ns}$ \\
$\mathrm{HDL}<40 \mathrm{mg} / \mathrm{dl}$ & $\mathrm{ns}$ & $\mathrm{ns}$ & $\mathrm{ns}$ \\
Tabaquismo & $\mathrm{ns}$ & $\mathrm{ns}$ & $\mathrm{ns}$ \\
$\mathrm{HVI}$ & $* * *$ & $* * *$ & $* * *$ \\
Proteinuria $>300 \mathrm{mg} / 24$ horas & $* * *$ & $* * *$ & $* *$ \\
Creatinina 1,2-2,0 mg/dl & $* * *$ & $* * *$ & $* * *$ \\
Retinopatía I-II & $* * *$ & $* * *$ & $* * *$ \\
AFP & + & $\mathrm{ns}$ & $\mathrm{ns}$ \\
Cardiopatía isquémica & $* *$ & $* *$ & $\mathrm{~ns}$ \\
Enf. cerebrovascular & $* * *$ & $* * *$ & $* *$ \\
Nefropatía diabética & $* * *$ & $* * *$ & $* * *$ \\
Enf. vascular periférica & $* * *$ & $* * *$ & $* * *$ \\
Retinopatía grave & $\mathrm{ns}$ & $* *$ & $\mathrm{~ns}$ \\
Peso & $\mathrm{ns}$ & $*$ & $\mathrm{~ns}$ \\
Talla & $\mathrm{ns}$ & + & $*$ \\
Edad & $* * *$ & $* * *$ & $* * *$ \\
IMC & $\mathrm{ns}$ & $\mathrm{ns}$ & $\mathrm{ns}$ \\
\hline
\end{tabular}

ns: $p>0,100 ;+: p<0,100 ;{ }^{*}: p<0,050 ;{ }^{* *}: p<0,010 ;{ }^{* * *}: p<0,001$. 
nopatía I-II, enfermedad cerebrovascular, nefropatía diabética, enfermedad vascular periférica y edad: La presencia de ateromatosis se presenta con una edad de media de 6 años mayor que la población en la que no se evidencia ateromatosis. Correlacionada en varones y en serie global con cardiopatía isquémica. En serie global con el sexo: Los hombres presentan más ateromatosis que las mujeres. En varones con retinopatía grave y peso. Con el peso de forma inversa, de tal manera que existe una diferencia de $2 \mathrm{~kg}$ de menos en la población ateromatosa. En mujeres con la talla, y de forma inversa. Un $17 \%$ de varones y un $10 \%$ de mujeres presentan ateromatosis.

\section{ENFERMEDAD VASCULAR PERIFÉRICA (EVP)}

- Correlacionada en serie global y en cada sexo con cifras altas de creatinina, ateromatosis, retinopatía I-II y cardiopatía isquémica. Correlacionada en varones y en serie global con HVI, proteinuria, cardiopatía isquémica, enfermedad cerebrovascular, nefropatía diabética, retinopatía grave, edad, IMC y peso. Con estos últimos parámetros de forma inversa. En serie global con tabaquismo y talla. En serie global y en mujeres con AFP.Un 9\% de varones y un 3\% de mujeres presentan enfermedad vascular periférica.

\section{ENFERMEDAD CEREBROVASCULAR}

Correlacionada en serie global con ateromatosis, edad y peso. En varones y en serie global con nefropatía diabética, EVP, retinopatía I-II e IMC. Con este último parámetro de forma inversa. Sólo en varones con niveles elevados de colesterol total alto y tabaquismo. Con tabaquismo se relaciona de forma inversa. Correlacionada sólo en mujeres con cifras bajas de colesterol HDL, y de forma inversa. Un 16,8\% de varones y un $10,7 \%$ de mujeres presentan enfermedad cerebro vascular.

\section{RETINOPATÍA GRAVE}

- Correlacionada en la serie global y en cada sexo con proteinuria, retinopatía I-II y nefropatía diabética. En varones y en serie global con HVI, niveles elevados de creatinina, y enfermedad vascular periférica. Correlacionada en serie global con la cardiopatía isquémica. Sólo en varones con ateromatosis y AFP de eventos cardiovasculares.

\section{NEFROPATÍA DIABÉTICA}

- Correlacionada en la serie global y en cada sexo con HVI, niveles elevados proteinuria y de creatinina, ateromatosis, retinopatía I-II, cardiopatía isquémica y retinopatía grave. En varones y en serie global con enfermedad cerebrovascular, enfermedad vascular periférica, cifras elevadas de colesterol total y edad. En serie global con la talla. Sólo en varones con niveles elevados de colesterol total. Un $6 \%$ de varones y un $2,67 \%$ de mujeres presentan nefropatía diabética.

\section{PESO}

- Correlacionado en serie global y en cada sexo con la talla, IMC, EFP, enfermedad cerebrovascular y edad. Con esta última de forma inversa, es decir aquellos pacientes que presentan un mayor peso son los de menor edad. Correlacionado en serie global y en varones con cifras elevadas de colesterol total y EFP. Con ella de forma inversa. En serie global con tabaquismo. Sólo en varones niveles elevados de colesterol LDL, ateromatosis, y cifras elevadas de creatinina. Con este último parámetro se correlaciona de forma inversa. Correlacionado en serie global y en mujeres con niveles bajos de colesterol HDL. Sólo en mujeres con la cardiopatía isquémica.

\section{TALLA}

-Correlacionada en serie global y en cada sexo con niveles bajos de colesterol HDL, tabaquismo, peso, IMC y edad. En serie global y varones con niveles altos de colesterol LDL y AFP de eventos cardiovasculares. En la serie global con proteinuria, niveles elevados de creatinina y EFP. Sólo en mujeres con ateromatosis, y de forma inversa.

\section{EDAD (Tabla V)}

- Correlacionada en serie total y en cada sexo con niveles elevados de creatinina, ateromatosis, cardiopatía isquémica, enfermedad cerebrovascular. De forma negativa o inversa está correlacionado con niveles de colesterol LDL elevado, tabaquismo, AFP de eventos cardiovasculares, peso, talla e IMC. La consulta médica es una media de cinco años más precoz que los pacientes que no tienen afectadas estas variables. En serie global y en varones con nefropatía diabética y EFP.De forma inversa está correlacionada con cifras elevadas de colesterol total. En la serie global y de forma inversa con el sexo y niveles bajos de colesterol HDL. Sólo en mujeres con HVI y proteinuria.

TABLA V

PERFILES DE ASOCIACIÓN DE LA VARIABLE EDAD

\begin{tabular}{lccc}
\hline Edad & & Varones & Mujeres \\
\hline Sexo & $* * *$ & $\mathrm{~ns}$ & $\mathrm{~ns}$ \\
Colesterol total $>250 \mathrm{mg} / \mathrm{dl}$ & $* * *$ & $* * *$ & $\mathrm{~ns}$ \\
$\mathrm{LDL}>130 \mathrm{mg} / \mathrm{dl}$ & $* * *$ & $* * *$ & $*$ \\
$\mathrm{HDL}<40 \mathrm{mg} / \mathrm{dl}$ & $* *$ & $\mathrm{~ns}$ & $\mathrm{~ns}$ \\
Tabaquismo & $* * *$ & $* * *$ & $* * *$ \\
$\mathrm{HVI}$ & + & $\mathrm{ns}$ & $* *$ \\
Proteinuria $>300 \mathrm{mg} / 24$ horas & $\mathrm{ns}$ & $* *$ & \\
Creatinina 1,2-2,0 mg/dl & $* * *$ & $* * *$ & $* * *$ \\
Ateromatosis & $* * *$ & $* * *$ & $* * *$ \\
Retinopatía l-Il & $\mathrm{ns}$ & $\mathrm{ns}$ & $\mathrm{ns}$ \\
AFP & $* *$ & $*$ & $*$ \\
Cardiopatía isquémica & $* * *$ & $* * *$ & $* * *$ \\
Enf. cerebrovascular & $* * *$ & $* * *$ & $* * *$ \\
Nefropatía diabética & $* *$ & $* *$ & $\mathrm{~ns}$ \\
Enf. vascular periférica & $* * *$ & $* * *$ & + \\
Retinopatía grave & $\mathrm{ns}$ & $\mathrm{ns}$ & + \\
Peso & $* * *$ & $* * *$ & $* * *$ \\
Talla & $* * *$ & $* * *$ & $* * *$ \\
IMC & $* * *$ & $* * *$ & $* * *$ \\
\hline
\end{tabular}

ns: $p>0,100 ;+: p<0,100 ;{ }^{*}: p<0,050 ;{ }^{*}: p<0,010 ;{ }^{* *}: p<0,001$. 
Respecto al sexo, los varones tienen respecto a las mujeres un mayor porcentaje de cifras de colesterol HDL bajo, tabaquismo, proteinuria de más de $300 \mathrm{mg} / 24$ horas, niveles de creatinina elevados, ateromatosis, cardiopatía isquémica, enfermedad cerebrovascular, nefropatía diabética, enfermedad vascular periférica, así como mayor peso: $6,7 \mathrm{~kg}$ de media, mayor talla: $11,5 \mathrm{~cm}$ de media y una edad más joven de casi 3 años de media en acudir a consultas.

$I M C$

- Correlacionado en cada sexo y en la serie total con peso, talla y edad. Con la edad de forma negativa. Correlacionado en serie total y en varones con cifras elevadas de colesterol total. De forma negativa lo está con el tabaquismo, EFP y niveles elevados de creatinina. Sólo en varones con retinopatía I-II. Correlacionado sólo en mujeres con cardiopatía isquémica.

\section{DISCUSIÓN}

La hipercolesterolemia es uno de los principales factores de riesgo modificables de la enfermedad cardiovascular. El estudio Multiple Risk Factor Intervention Trial (9) demostró la existencia de una relación entre la hipercolesterolemia y la mortalidad total por cardiopatía isquémica. La reducción de la colesterolemia lleva consigo una disminución de la incidencia y mortalidad por cardiopatía isquémica y enfermedad cardiovascular en general, tanto en la prevención primaria como en la secundaria (10).

Un estudio nacional utilizando técnicas de química seca, encontró que un $18 \%$ (18,6\% de los varones, y el 17,6\% de las mujeres) de la población española de 35 a 64 años, tenía una colesterolemia igual o superior a $250 \mathrm{mg} / \mathrm{dl}$ y un $57 \%$ superior a $200 \mathrm{mg} / \mathrm{dl}$. En nuestro estudio también hemos encontrado un mayor porcentaje de varones que de mujeres que tienen cifras de colesterol total por encima de 250 $\mathrm{mg} / \mathrm{dl}$. Epidemiológicamente la asociación entre la hipercolesterolemia y otros factores de riesgo cardiovascular es frecuente. Así Banegas observa un gradiente ascendente en las cifras medias de presión arterial, relacionado con el aumento de las cifras de colesterol sérico (11). En un estudio realizado en Atención Primaria se observó que en un $31 \%$ de los pacientes de 35 a 65 años que acuden a la consulta del médico de familia, tienen dos factores de riesgo cardiovascular y un $6 \%$ tres factores de riesgo, considerando los hipertensión, la hipercolesterolemia y el tabaquismo (12). En población anciana es frecuente la asociación de varios factores de riesgo (13). Especial riesgo cardiovascular conlleva la dislipemia en los diabéticos (14). Nuestros hallazgos también correlacionan la cifra de colesterol total y LDL elevados, especialmente en varones, con la cardiopatía isquémica, tabaquismo, retinopatía, enfermedad cerebrovascular, nefropatía diabética, AFP de eventos cardiovasculares y peso del paciente. Las cifras de HDL bajo también se encuentran correlacionadas en nuestra casuística con tabaquismo, cardiopatía isquémica y enfermedad cerebrovascular en los dos sexos. Es especialmente reseñable como el tabaquismo, en los dos sexos, se encuentra correlacionado con una alteración del espectro lipídico global.
Diversos estudios prospectivos, entre ellos el estudio Framingham, han mostrado un ascenso del riesgo de mortalidad total y cardiovascular al ir aumentando los niveles de presión arterial (diastólica y sistólica) siendo esta relación continua y gradual $(15,16)$. Como en otros países también en España es significativamente mayor la prevalencia de hipercolesterolemia, hipertrigliciridemia, diabetes mellitus, tabaquismo y obesidad en la hipertensión que en la no hipertensión (17), y además estos factores son también mal controladas como la propia HTA $(18,19)$. Los pacientes con un primer episodio coronario tienen una probabilidad cinco veces mayor de presentar hipertensión que en pacientes sin enfermedad coronaria previa, seguido de obesidad y de dislipemia (20) más del 70\% de los hipertensos presentan otros factores de riesgo cardiovascular $(21,22)$. Se estima que en la población de España de edades medias, el $42 \%$ de las muertes por enfermedades cardiovasculares y el $25,5 \%$ de las muertes totales están relacionadas con la hipertensión (cifras de $>0=$ de 140/90 $\mathrm{mmHg}$ ). En términos absolutos ello supone unas 4000 muertes cardiovasculares y unas 14.000 muertes totales al año relacionadas con la hipertensión (23).

Las estrategias sobre el manejo de la hipertensión no deberían ser tomadas únicamente por la elevación de las cifras tensionales, sino por la presencia de otros factores de riesgo cardiovascular asociados, lesión de órgano diana y otras condiciones clínicas asociadas (17). Es conocido que hay otros factores de riesgo no clásicos que también han demostrado tener una relación con la enfermedad cardiovascular, y que no se tienen en cuenta en el cálculo de riesgo cardiovascular, como la obesidad, el sedentarismo, historia familiar de enfermedad coronaria temprana o de eventos cardiovasculares en general, lipoproteínas de baja densidad (LDL) pequeñas y densas, lipoproteína a, fibrinógeno, homocisteina, factores inflamatorios, psicosociales, laborales y probablemente otros que deben de considerarse dentro del contexto clínico de cada paciente (24-26).

La presencia de una historia familiar precoz cardiovascular, se encuentra relacionada con la presencia de otros factores de riesgo cardiovascular en el paciente (27-29). En un anterior estudio de 2.264 pacientes procedente del Estudio CIFARC (Grupo de Riesgo Vascular de la SEMI), encontramos una asociación significativa entre los diferentes factores de riesgo cardiovascular o lesión de órganos diana y los AFP de enfermedad cardiovascular, preferentemente en hombres. Observamos como los pacientes varones con AFP acudían una media de cinco años antes a consultas que los que carecían de dichos antecedentes. En las mujeres con AFP ese adelanto era de siete años (30). Se ha descrito un incremento continuo y gradual del riesgo relativo de mortalidad, conforme aumenta el índice de masa corporal (31). Se ha observado además una asociación del IMC y la obesidad con la hipertensión arterial, hiperglucemia y el sedentarismo (32-34), incluso en niños y adolescentes, pudiendo existir también una correlación con historia familiar positiva a enfermedad cardiovascular. más específicamente, algunos factores de riesgo cardiovascular tienden a agregarse o a formar un cluster, debido a que están metabólicamente ligados (obesidad, diabetes, hipertensión y dislipemia, que constituyen el llamado síndrome metabólico o síndrome de resistencia a la insulina que puede llegar a ser muy frecuente en las edades medias y avanzadas de la vida 
(35-37). En nuestro estudio también hemos encontrado una correlación positiva de IMC en hombres con niveles elevados de colesterol, HVI y retinopatía leve. En mujeres se ha encontrado una correlación positiva con la cardiopatía isquémica.

Según la Encuesta Nacional de Salud en 1997, la frecuencia de obesidad es mayor en varones jóvenes que en mujeres jóvenes, pero a partir de los 45 años se hace más frecuente en las mujeres que en los hombres. En ambos sexos la prevalencia de obesidad aumenta con la edad $(19,2 \%)$ en el grupo de 45 a 64 años y $21,2 \%$ en el grupo de 65 a 74 años. Por otro lado, datos de peso y talla medidos en sujetos de 35 a 64 años del Proyecto Mónica de la OMS, sitúan a España (prevalencia de obesidad de $16 \%$ en varones y $25 \%$ en mujeres) en un lugar intermedio a mediados de los años noventa (38). Otro factor de riesgo cardiovascular importante es la HVI que traduce la afectación de órganos diana en varones. La población hipertensa de España se ha estimado en un 64\%; la presencia de HVI diagnosticada con ecocardiografía ha sido del 40\% (39). Hemos observado un perfil positivo de asociación de la HVI con AFP de eventos cardiovasculares y afectación de otros órganos diana. Respecto a la proteinuria, hemos observado que ésta, en ambos sexos, se encuentra íntimamente correlacionada con niveles elevados de creatinina, HVI y retinopatía leve y grave, como traducción de la afectación de los órganos diana por la HTA. En mujeres está asociada a niveles bajos de colesterol HDL. Hemos evidenciado también que niveles elevados de creatinina, retinopatía leve y grave, se correlacionan con otros factores de riesgo cardiovascular y afectación de órganos diana. La diabetes mellitus como ya mostró el Estudio Framingham se asocia a un mayor riesgo de desarrollo de enfermedad cerebrovascular (40). De hecho las complicaciones de la ateromatosis son la principal causa de morbimortalidad en los diabéticos. Su riesgo relativo de enfermedad cardiovascular es mayor en la mujer diabética que en el varón, con cerca de 10.000 defunciones por año. Supone la sexta causa de muerte en España (42,6\% de todas las defunciones) según el Instituto Nacional de Estadística en el 2002 (41). Nosotros también en ambos sexos la hemos encontrado asociada a un mayor riesgo cardiovascular y afectación de órganos diana. La cardiopatía isquémica se encuentra íntimamente relacionada con otros factores de riesgo cardiovascular como el tabaco, sedentarismo e hipertensión arterial. En los varones a estos factores, se les puede atribuir aproximadamente la mitad de las muertes coronarias en los años noventa. En las mujeres, son el sedentarismo, la hipertensión y la hipercolesterolemia, los tres factores que provocan una mayor carga coronaria (42). En el Estudio Ibérica los pacientes con infarto de miocardio presentaban en el momento del ingreso hospitalario una prevalencia de hipertensión del $46 \%$, tabaquismo $43 \%$, dislipemia $39 \%$ y de diabetes del 18\%. El $32 \%$ tenían dos o más factores de riesgo, y sólo un $18 \%$ no presentaban estos factores de riesgo (43). En otros estudios (44) donde se evaluó el perfil de infarto de miocardio en adultos jóvenes entre 24 y 40 años, se encontró que el 75\% presentaban tensión emocional por personalidad de tipo A, el $71 \%$ tabaquismo, $42 \%$ hipertensión arterial sistémica, 25\% diabetes mellitus, $25 \%$ hiperlipemia y el $20 \%$ obesidad. Un $24 \%$ no presentaban factor de riesgo alguno conocido.

En un $98 \%$ de los casos se encontró presente por lo menos un factor de riesgo familiar. En otro estudio que se valoró la presencia de un infarto en pacientes muy jóvenes de menos de 30 años (45) encontró que muy pocos de estos pacientes tenían factores de riesgo habituales; por el contrario se trataba de pacientes muy fumadores la mayoría. Un $50 \%$ tenían normales las coronariografías, mientras que en otro $50 \%$ evidenciaron signos de enfermedad coronaria. Los autores apuntan que una tendencia trombofílica y un vasoespasmo deberían de ser considerado en estos casos. En el Estudio Prevese (46) se evidenció a los seis meses del alta tras infarto agudo de miocardio, una mejoría del perfil de riesgo cardiovascular, aunque no hubo modificaciones favorables en las concentraciones del colesterol total. Tras cuatro años después de un infarto, el Estudio Prevese II, encontró una disminución significativa del tabaquismo $(41,6 \%$ frente al $35,4 \%)$ y detectó la alta prevalencia de hipertensión $(47,5 \%)$ sobrepeso $(46,3 \%)$ y obesidad $(48 \%)$ (47). Nosotros también hemos visto la cardiopatía isquémica asociada con factores de riesgo cardiovascular, otras enfermedades vasculares y afectación de órganos diana. Por lo que se refiere a la enfermedad cerebrovascular, tanto en los varones como en las mujeres, la hipertensión arterial es el factor con mayor impacto potencial seguido de la hipercolesterolemia. El impacto de ésta sería mayor si se considerasen cifras de más de $200 \mathrm{mg} / \mathrm{dl}$ en vez de $250 \mathrm{mg} / \mathrm{dl}$. La obesidad tendría también un mayor impacto si considerásemos el incremento ocurrido en los últimos años. El sobrepeso está asociado también a riesgo cardiovascular (48). Se ha visto que altos niveles de homicisteina se encuentran correlacionados con la severidad del accidente cerebrovascular, y que en familias con este historial los niveles en niños están determinados por lo de los padres (49). La morbilidad por enfermedad isquémica del corazón en los varones, es casi el doble que por enfermedad cerebrovascular, mientras que en las mujeres la morbilidad cerebrovascular es ligeramente superior a la isquémica. En estos últimos años la enfermedad isquémica del corazón ha aumentado más que la cerebrovascular. Nosotros hemos visto asociada la enfermedad cerebrovascular, además de la hipercolesterolemia, con la ateromatosis, enfermedad vascular periférica y el tabaquismo. En un estudio Bergmark comprueba como los pacientes con enfermedad vascular periférica presentan niveles incrementados de autoanticuerpos contra el colesterol LDL oxidado. Además la presencia de hipertensión y una historia familiar de eventos cardiovasculares eran los únicos factores asociados de forma significativa con dichos incrementados niveles de autoanticuerpos (50). Valentine y cols. (51) en otro estudio, comprueban como una historia familiar de eventos cardiovasculares es el mayor determinante de enfermedad arterial oclusiva periférica subclínica en jóvenes pacientes adultos. Además encontró una alta proporción de grandes fumadores en estos pacientes, donde se encontraban lesiones claramente detectables aunque todavía asintomáticas. Una historia familiar y tabaquismo se comportaron como factores aditivos en la enfermedad vascular periférica. Otros factores que encontraron también implicados fueron la hipercolesterolemia, hiperhomocisteinemia, estados de hipercoagubilidad y elevados niveles de lipoproteina a. Así mismo evidenciaron que la mayoría de los pacientes con enfermedad arterial oclusiva precoz tenían también enfermedad coronaria.

Diversos autores como Van Den Bosh y Collheri $(52,53)$ han encontrado una asociación positiva entre el consumo de 
anticonceptivos y la enfermedad vascular periférica, aunque observaron un efecto protrombótico más agudo que por mecanismo de actuación de tal forma que condujeran a una progresión de ateroesclerosis como fenómeno crónico. No obstante tal asociación no es frecuente, aunque el riesgo aumenta de 100 a 200 veces si la paciente es fumadora. Aconsejan discontinuar el uso de anticonceptivos. Autores como Ridker y cols. (54-55) han relacionado con la enfermedad vascular periférica a nuevos factores de riesgo, como niveles aumentados de homocisteina, infecciones crónicas, niveles elevados de Proteina $\mathrm{C}$ reactiva. Nosotros la hemos encontrado correlacionada con el tabaquismo y la cardiopatía isquémica, así como con otras enfermedades vasculares y factores de riesgo cardiovascular, observándose además un incremento con la edad de los individuos. Wang y cols. (56) encuentran con estudios ultrasónicos, un engrosamiento a nivel de la íntima media de carótida interna, muy prevalente en pacientes con historial familiar precoz, antes de los 60 años, de enfermedad coronaria. Este substrato anatomopatológico de ateroma subclínico puede traducir una predisposición familiar para enfermedad cardiovascular. Apunta Wang que este engrosamiento de las carótidas puede constituir un fenotipo fácil que exprese una transmisión genética de enfermedad cardiovascular. Según Barker (57), la ateroesclerosis puede considerarse con una visión longitudinal a lo largo de la vida. Según esta visión la enfermedad cardiovascular se condiciona genéticamente en el momento de la concepción, y se expresa por interacciones con los factores del medio ambiente que ya actúan en etapas tan tempranas de la vida como la perinatal y la primera infancia. La malnutrición en estos periodos conduce a retrasos de crecimiento y en la primera infancia es posible que "programen" alteraciones metabólicas-fisiológicas, para aprovechar mejor los nutrientes escasos que aumentan la susceptibilidad a la enfermedad en la época adulta. Este mantenimiento de condiciones adversas de la vida, también puede acompañarse de adquisición de hábitos de vida no favorables para la salud en la adolescencia, como el tabaquismo, consumo excesivo de alcohol, dieta inadecuada, sedentarismo, que van conduciendo progresivamente a la enfermedad cardiovascular en la vida adulta (59). Nosotros la hemos encontrado asociada en ambos sexos con factores de riesgo cardiovascular, afectación de órganos diana, y enfermedad cardiovascular. En varones, al consumo de tabaco, sedentarismo e hipertensión se pueden atribuir aproximadamente la mitad de las muertes coronarias ocurridas en los años noventa (59).

Autores como Valentine (60) han encontrado una fuerte asociación entre el hábito de fumar y un padecimiento precoz de la enfermedad vascular periférica. La ateroesclerosis se desarrolla en arterias periféricas incluso en ausencia de otros factores de riesgo tales como una historia familiar de diabetes o de hipertensión. En el Estudio Yuth, fumar estaba asociado con más extensas estrías grasas y elevadas lesiones de la aorta abdominal, en personas entre 15 y 34 años de edad que murieron por otras causas (61). Nosotros hemos evidenciado una correlación significativa entre tabaquismo y enfermedad vascular periférica, únicamente cuan- do hemos analizado la casuística de forma global, es decir hombres y mujeres juntos. Así mismo hemos observado su asociación con otros factores de riesgo cardiovascular y AFP de eventos cardiovasculares. Como otros autores, también hemos encontrado diferencias entre sexo y edad con diferentes factores de riesgo vascular, observando un incremento de enfermedad cardiovascular y afectación de órganos diana con la edad. En España mueren más mujeres que varones por enfermedad cardiovascular. De las 131.774 muertes cardiovasculares que se produjeron en 1999, el $55 \%$ se dieron en mujeres, y el $45 \%$ en varones. Si se ajusta la mortalidad por edad, ésta es más alta en los varones que en las mujeres (tasas de mortalidad ajustada son un $40 \%$ mayores en los varones que en las mujeres en España). Las tasas por cardiopatía isquémica son en torno al doble en varones que en mujeres. Esta situación paradógica en que las tasas de mortalidad específicas de edad son mayores en los varones, pero la mortalidad proporcional, las tasas brutas de mortalidad y el número absoluto de muertes, son superiores en las mujeres, es el resultado de hechos bien conocidos (62). Hay un mayor riesgo cardiovascular de los varones reflejado en las tasas específicas de edad. De hecho el riesgo coronario de los varones es similar al de las mujeres que son 10 años mayores que aquellos. La enfermedad cardiovascular es más frecuente en las edades más avanzadas de la vida, donde el número de mujeres es muy superior al de los varones. Ello explica que las mujeres, a pesar de tener menor riesgo cardiovascular que los varones, tengan mayor mortalidad proporcional bruta y número de muertes por esta causa (63). Razones similares explican por qué la mortalidad proporcional por ictus es mayor en las mujeres que en los varones. Las mujeres poseen un menor riesgo coronario que los varones, y por tanto una mayor probabilidad de llegar a edades avanzadas en los que la mortalidad por ictus es más frecuente. A este respecto Marrugat (64) comprueba que el infarto de miocardio presenta un pronóstico más severo y más letal en las mujeres que en los varones. En otro estudio Vogels (65) comprueba como la morbilidad tanto para el angor pectoris como para el infarto de miocardio, es mayor en mujeres con un nivel socioeconómico bajo. Respecto a la edad, las tasas de mortalidad cardiovascular aumenta enormemente a medida que se incrementa la edad, siendo superior a mil por 100.000 habitantes en las personas mayores de 70 años, siendo la primera causa de muertes a esta edad; situándose en siguiente posición detrás de los tumores en edades más jóvenes (62).

Como conclusión de este estudio podemos afirmar que existen diferentes perfiles asociativos entre las variables estudiadas.

\section{AGRADECIMIENTOS}

Al grupo de Investigadores CIFARC del Grupo de Riesgo Cardiovascular de la Sociedad Española de Medicina Interna que recogieron los datos estadísticos gracias a lo cual se ha podido elaborar este estudio. 


\section{Bibliografía}

1. Villar Alvárez F, Banegas Banegas JR, De Mata Donado Campos J, Rodríguez Artalejo F. Los factores de riesgo en España. Hechos y Cifras. Madrid: Ergon; 2003.

2. Martínez de Aragón MU, Llacer A. Mortalidad en España 1997, Boletín Epidemiológico General 2000; 8: 253-69.

3. Marrugat J, Elosua R, Martí H. Epidemiología de la cardiopatía isquémica en España:Estimación de número de casos y de las tendencias entre 1997 y 2005. Rev Esp Cardiol 2002; 55: 331.46.

4. Pérez G, Pena A, Sala J, Roset PN, Masia R, Marrugat J and the Regicor Investigators. Acute myocardial infarction care fatality, incidence and mortality rates in a population registry in Gerona, Spain. Int J Epidemiol 1998; 27: 599-604.

5. Tunstall Pedoe H, Kuulasmik K, Mähönem M, Tolonen H, Ruokokoshi $\mathrm{E}$, Amougel $\mathrm{P}$, for the Monia Proyect Contribution of trends in survival and coronary event rates to charges in coronary heart disease mortality.10 year results from 37 Vho Monica proyect populations. Lancet 1999, 353: 1547-57.

6. Would Health Organization. The Wordl Health Report 2002; Risks to Health 2002. Geneva: Wordl Health Organization.

7. 2003 Wordl health organization Society of Hipertension. Statemant on management of hypertension. Would Health Organization International Society of Hypertension Writing Grouyp. J Hypertens 2003; 21: 19831992.

8. Fiol M, Cabads A, Sala J, Marrugat J, Elosua R, Vega G, et al. Variabilidad en el manejo hospitalario del infarto de miocardio en España. Estudio Ibérica. Cardiopatía Isquémica 2001; 4: 443-452.

9. Multiple Risk factor Intervention Trial Research Group. Multiple risk factor intervention trial. Risk Factor Changes and mortality results. Jama 1982; 248: 1465-1477.

10. Plaza Pérez I, Villar Alvárez F, Mata López P, Pérez Jiménez F, Márquez Galán A, Casasnovas Lenguas JA et al. Control de la colesterolemia en España 2000. Un instrumento para la población cardiovascular. Rev Esp Cardiol 2000; 53: 815-837.

11. Banegas JR, Villar F, Pérez C, Jiménez R, Gil E, Muñiz J, Juane R. Estudio epidemiológico de los factores de riesgo cardiovascular en la población española de 35 a 64 años. Rev San Hig Pub 1993; 67: 419445.

12. Maiques A, Morales MM, Franch M, Alfonso MD, Moreno Manzanero P; García JM. Cálculo de riesgo coronario de los pacxientes incluidos en el Programa de Actividades Preventivas y de Promoción de la Salud. Aten Primaria 1995; 15: 86-92.

13. Gil B, Avilés J, Maldonado A, Fernández A. Factores de riesgo en ancianos. Un estudio de 143 pacientes. An Med Interna (Madrid) 1997; 14: 495-499.

14. González P, Herrera J, Ascasso JF, Escobar F, Gómez Genique JA, Jiménez Perepérez JA, et al. Dislipemia diabética: Documento de consenso de la Salud española de Diabetes y la Sociedad Española de Arteriosclerosis. Av Diabetol 1998; 14: 33-43.

15. Stamler J, Neaton JD, Wentoworth DN. Blood pressure (systolic and diastolic) and risk of total coronary hearth disease. Hypertension 1989; 13 (5 Supl): 12-112.

16. Machmohon S, Peto R, Cutler J, et al. Blood pressure stroke and coronary heart disease. Part 1. Prolonged differences in blood pressure: prospective observational studies corrected for the regression dilution bias. Lancet 1990; 335: 765-774.

17. 2003 Wordl Health Organization (WHO9 International Society of Hypertension (ISH) Statement on nanagement of hypertension. J Hypertension 2003; 21: 1983-1992.

18. Godley P, Pham H, Rohack J, Noodward B, Yokogame K, Mane JK, Opportunities for improving the quality of hypertension care in a managed care setting. Am J Helath Syst Pharm 2001; 58: 1728-1773.

19. Trilling JS, Froom J. The urgent need improve hypertension care. Arch From Med 2000;9: 794-801.

20. Brotons C, Cascant P, Ribera A, Moral I, Gaieté-Permanger. Utilidad de la medición del riesgo coronario a partir de la ecuación del Estudio Framingham: Estudio de casos y de controles. Med Clin (Barc) 2003; 121 (9):327-330

21. Mosquera JD, Brec AJ, Rumalle Gómara E, Gómez C, Márquez M, Sanz M. Prevalencia de los factores de riesgo cardiovascular en población adulta de Logroño, La Rioja. Clin invest Arterioscl 2000; 12: 199-208.

22. Segura A, Rius G. Factores de riesgo cardiovascular en una población rural de Castilla La Mancha. Rev Esp Cardiol 1999; 52: 577-588.

23. Banegas JR, Rodríguez Artolejo F, Cruz JJ, De Andrés B, Rey Calero J.
Mortalidad relacionada con la presión arterial y la hipertensión en españa. Med Clin (Barc) 1999; 112: 489-494.

24. Saitta A, Castaldo M, Sardo A, Linquegrani M, Bonaiuto M, Zema M, et al. Elevated levels of lipoprotein(a) are present in subjects with early ischemic cardiopaty and with familiar history of ischemic cardiopathy. Minerva Med 1999; 90 (5-6): 151-8.

25. Torbus-Lisiecka B, Bukowska H, Jastrzebska M, Chelstowski K, Honezarenko K, Nuruszewicz M. Lp (a) homocysteine and familiy history of early ischemic cerebral stroke. Nutr Metab Cardiovase Dis 2001; 11 (5): 52-9.

26. Ortega RM, Jiménez A, Andrés P, Faci M, Lolo JM, Lozano MC, et al. Homocysteine levels in elderly Spanish people influence of pyridoxine vitamin B12 and folic acid intakes. J Nutr Health Aging 2002; 6: 69-71.

27. Friedlander Y, Siscovick DS, Weinmann S, Austin MA, Plasty BM, Lemaitre RN, et al. Family history as a risk factor for primary cardiac arrest. Circulation 1998; 97:155-160

28. James Valentine R, Guerra R, Stephan P, Sloggins E, Clagett P, Cohen $\mathrm{J}$. Family history is mayor determinant of subclinical peripheral arterial disease in joung adults. J Vasc Surg 2004; 39: 351-6.

29. Jerrad-Dunne P, Markus HS, Steckel DA et al. Early carotid atheromatosis and family history of vascular disease:specific effects on arterial sites have implications for genetic studies. Arterioscler Thromb Vasc Biol 2003; 23: 302-306

30. Ebrí Torné B, Ebrí Verde MI, Portolés Suso A, Pérez Conesa A, Fuentes Solsona F. Conde JI. Diferencias entre variables representativas de enfermedad o repercusión cardiovascular entre sujetos de alto riesgo cardiovascular con y sin antecededentes familiares de eventos cardiovasculares precoces. An Med Interna (Madrid) 2006; 23: 11-18.

31. Manson JE, Wuillet WC, Stampher MJ, Body Weifht and mortality among women. N Engl J Med 1995; 333: 677-685.

32. U.S. Department of Health and Human Services. The Surgem General"s Report on Nutrition and Human Services. Public Health Service 1988.

33. Wordl Health Organization Obesity: Preventing and managing the global epidemic. Ginebra: Who Technical report Series, na 894, 2000.

34. Glowinska B, Urban M, Koput A. Correlation between body mass index, lipoprotein (a) level positive family history of cardiovascular disease in children and adolescent with obesity, hypertension and diabetes. Pol Merkuriusz Lek 2002; 12 (68): 108-14.

35. Ford Es, Giles NH, Dietz WH. Prevalence of the metabolic syndrome among us adults. Findings from the Third National health and Nutrition examination Survey. Jama 2002; 287: 356-359.

36. Rabkin SW, Chen Y, Leiter L, Liu L, reeder BA. Risk factor correlates of body mass index. Canadian Hearth health Survey Research Group. CMAJ 1997; 157: 526-531

37. Siervogel RM, Wisemandle W, Maynard LM, Gui SS, Chemlea WC, Towne B. Lifetime owerweight status in relation to serial changes in body composition and risk factors for cardiovascular disease: The Fels Longitudinal Study. Obes Res 2000; 8:422-430.

38. Kuulasman K, Tunstall-Pedae H, Dobbon A, Fortmam S, Saqm S, Tolomen H, et al., Tuohmiletto J, for the Who Monica Proyect. Estimation of contribution of changes in classical risk factors to trends in coronary event rates across the Who Monice. Proyect populations. Lancet 2000; 355: 675-687.

39. Dalfo A, Bayo J, Gil M, Campillo M, Botej A, Vida MA, et al. Hipertrofia ventricular izquierda en población general hipertensa de Barcelona. Med Clin (Barc) 1995; 105:361-366.

40. Kannel WB, MacGee DI. Diabetes and cardiovasc risk factors: the Framingham Study. Circulation 1979; 59:8-13.

41. Instituto Nacional de Estadística. Defunciones según la causa de muerte, 1991. Madrid: Instituto nacional de Estadística 2002.

42. Banegas JR, Rodríguez Artalejo F. El problema de la hipertensión arterial en España. Rev Clin Esp 2002; 202:12-15

43. Fiol M, Cabadés A, Sala J, Marrugat J, Elosua R, Vega G, et al., García $\mathrm{J}$, en representación de los investigadores del Estudio Ibérica. Variabilidad en el manejo hospitalario del infarto agudo de miocardio en España. Estudio Ibérica (Investigación, búsqueda específica y registro de isquemia coronaria aguda) Rev Esp Cardiol 2001;54:443-452.

44. Baduí E, Rangel A, Valdespino A, Graef A, Plaza A, Chávez E, et al. Infarto agudo de miocardio en adultos jóvenes. Presentación de 142 casos. Arch Inst cardiol Méx 1993; 63:529-537.

45. Gostmamm I, Cotan CH, Mosseri M. Clinical manifestations and outcome of acute myocardial infarction in very young patients. IMAJ 2003; 5: 633-636. 
46. Velasco JA, Cosín J, López Sendón JL, de Teresa E, Oya M, Carrasco JL, et al. La prevención secundaria del infarto agudo de miocardio en España. Estudio PREVESSE. Rec Clin Esp Cardiol 1997; 50: 406-415.

47. Velasco JA, Cosín J, López-Sendón JL, de Teresa E, Oya M, Seller G, en representación del Grupo de investigadores del Estudio PREVESSE II. Nuevos datos sobre la prevención secundaria del infarto agudo de miocardio en España. Resultados del estudio PREVESSE II. Rev Clin Esp Cardiol 2002; 55: 801-809.

48. Instituto Nacional de Estadística. Encuesta de Morbilidad Hospitalaria. Año 1999. Madrid: Instituto Nacional de Estadística, 2002.

49. Torbus Lisiecka B, Burkowska H, Jastrzebska M, Chelstowski K, Honczarenko K, Naruszewicz M. Lp (a), homocysteine and a family history of early ischemic cerebral stroke. Nutr Metab Cardiovasc Dis 2001; 5: 52-9.

50. Bergmark C, Un R, de Faire U, Lefvert Ak, Swedenborg J. Patients with early-onset peripheral vascular disease have increased levels of antibodies against oxidired LDL. Arterioscler Thromb Vasc Biol 1995; 15: 441-5,

51. Valentine J, Guerra R, Stephan P, Scoffins E, Clagett GP, Cohen J. Family history is a major determinant of subclinical peripheral arterise disease in yang adults. J Vase Surg 2004; 39: 351-6.

52. Van Den Bosh J, Kemmeren JM, Tanis BC, Mali M, Helmerhost FM, Rosendad FR, Añegra A, and Van der Graaf. The Ratio Study:oral contaceptives and the risk of peripheral arterial disease in young women. $\mathrm{J}$ Thrombos Haemost 2003, 1: 339-444.

53. Collheris and Cosmi B. Atherothombosis : What ratio (nale) for the ratio studies? J Thrombos Haemost 2003; 1: 418-420.

54. Ridker PM, Cushman M, Stampher MJ. Plasma concentration of C-reactive proteins and risk of developing peripheral vascular disease. Circulation 1998; 97: 425-428.

55. Ridkes PM, Stampher MJ, Riferi N. Novel risk factors for systemic atherosclerosis: a comparison of C-reactive proteins, fibrinogen. Homocystein, lipoprotein (a) and standard cholesterol screaning as predictors of peripheral arterial disease. Jane 2001; 285:2481-2485.

56. Wang TJ, Nam BJ, Dagostino RB, Wolf PA, Lloid-Jones DM, Macral
CA, et al. Carotid intima media thicknem is associated with premature coronary hear disease. The Framingham Heart Study. Circulation 2003; 108: 572-576.

57. Barken OSP. Fetal origine of coronary heart disease. BJM 1995; 311: 171-176.

58. Guallar-Castillón P, Rodríguez-Artalejo F, Banegas JR, De Andrés B, Rey Calero J. Factores ambientales en la vida temprana y nivel socioeconómico en la actualidad: ¿Cúal es más importante para la mortalidad cardiovascular en España?: Med Clin (Barc) 1999; 113: 444-446.

59. Banegas A, Banegas JR, Rodríguez Artalejo F, Graciani A. Epidemiología de la hipertensión arterial y mortalidad relacionada en España. En: De La Figuera M, Arnau JM, Brotons C. Hipertension arterial en atención primaria: evidencia y práctica clínica: Barcelona: Euromedical 2002.

60. Valentine RS, Kaplan HS, Green R, Jacobson DN, Myers SI, Clagett GP. Lipoprotein (a), homocystein and hypercogulable stars in young men with premature peripheral atherosclerosis: a perspective controlled analysis. J Vasc Surg 1996; 23:53-56.

61. The pathological determinants of atherosclerosis in fourth (PDAJ) Research Group. Relationship of atherosclerosis in young men to serum lipoprotein cholesterol concentrations and smoking. Jama 1990; 264: 301824.

62. Instituto Nacional de Estadística. Defunciones según la causa de muerte 1999. Madrid: Instituto Nacional de Estadística 2002.

63. Rodríguez Artalejo F, Banegas JR, Guallar-Castillón P. La mortalidad cardiovascular disminuye pero las muertes aumentan. Clin Invest Arteriosclerosis 2001; 13: 68-69.

64. Marrugat JM, Sala J, Masía R, Pavesi M, Sanz G, Valle V, et al., Elosua $\mathrm{R}$, for the Rescate Investigators. Mortality differences between men and women following first myocardial infarction. Jama 1998; 280: 14051409.

65. Vogels EA, Lagro-Janssen AL, Van Well C. Sex differences in cardiovascular disease: are women with low socioeconomic status at high risk? British Journal of General Practice 1999; 49: 963-6. 